\title{
Interface de recuperación para catálogos en línea con salidas ordenadas por probable relevancia
}

\author{
G ustavo G abriel A rchuby \\ Licenciatura en Informática, Estudiante. Facultad de Informática, \\ Universidad N acional de La Plata. \\ E-mail: gustavoa@ huma.fahce.unlp.edu.ar
}

\section{Julián C ellini}

A nalista en Computación, expedido por la Facultad de Informática de la U niversidad N acional de La Plata. Promedio: 7,625.

E-mail: juliancellini@gmx.net

\section{C laudia M arcela G onzález}

Bibliotecaria D ocumentalista, expedido por la Facultad de $\mathrm{H}$ umanidades y Ciencias de la Educación de la Universidad N acional de La Plata, 1988.

E-mail: claudia@huma.fahce.unlp.edu.ar

\section{Mónica Gabriela Pené}

Bibliotecaria D ocumentalista, expedido por la Facultad de H umanidades y Ciencias de la Educación de la U niversidad N acional de La Plata, con fecha 20 de diciembre de 1996. Promedio: 9,67 E-mail: mpene@ huma.fahce.unlp.edu.ar

\section{Resumen}

Se presenta el desarrollo de una interface de recuperación de información para catálogos en línea de acceso público (plataforma CDS/ISIS), basada en el concepto de similaridad para generar los resultados de una búsqueda ordenados por posible relevancia. Se expresan los fundamentos teóricos involucrados, para luego detallar la forma en que se efectuó su aplicación tecnológica, explícita a nivel de programación. Para finalizar se esbozan los problemas de implementación según el entorno.

\section{Palabras-claves}

Interfaces de recuperación de información; Ponderación de términos; Medida de similaridad; CDS/ISIS.

\section{Opacs retrieval interface with ranked outputs}

\begin{abstract}
Presents an information retrieval interface model for Public Access Catalogs (OPALS) on CDS/ISIS platform, based on the similarity principle. The proposal aims at ordering the results of vector queries according to their relevance. The underlying theoretical principle is described, as well as the implementation of the model.
\end{abstract}

\section{Keywords}

Information retrieval interfaces; Term weighting; Similarity measure; CDS/ISIS.

\section{INTRODUCCIÓN}

En estos últimos 50 años ha existido en los países desarrollados una preocupación constante por investigar e implementar técnicas que permitan recuperar información precisa. D esde mediados de siglo, los esfuerzos convergentes de distintas disciplinas: informática, lingüística, psicología, ciencias de la información, han dado origen a sistemas automáticos de recuperación de información de diferente nivel de complejidad. En el ámbito de la documentación, los más difundidos y utilizadosinternacional mente son los que aplican técnicas basadas en la equiparación exacta (exact matching), proximidad y álgebra de Booled.

Q uizá uno de los principales problemas de estos sistemas tradicionales, provenga de la falta de asignación de grados de posible relevancia en las respuestas². Esto es: el operador A N D es demasiado restrictivo, todos los documentos que no cumplen con las condiciones de búsqueda establecidas quedan excluidos; y en contraposición, el operador OR es demasiado inclusivo provocando generalmente un problema de sobrerecuperación. Por ejemplo, si al momento de plantear una estrategia de búsqueda sencilla, se opta por relacionar dostérminos con un A N D, el usuario perderá la posibilidad de ver los documentos que sólo contienen uno de los términos. Si la misma búsqueda se plantea con un $O R$, el sistema traerá todos los registros que contienen al menos uno, pero no es capaz de ordenar los registros recuperados de forma que se muestren primero los que contienen ambos términos.

El fundamento teórico que permitió desarrollar las técnicas de salidas ordenadas por probable relevancia (ranked output), correspondió al ámbito de la psicolingüística, concretamente a lostrabajos de Zipf. Sus estudios establecieron que si se ordenan las palabras de un corpus textual en un rango de forma descendente por su frecuencia de aparición, y luego se multiplica el rango por la frecuencia, se obtiene un valor aproximadamente constante. La observación de que dicho valor era más estable en las frecuencias intermedias, le permitió concluir que es en esas palabras donde se deposita la significación de un texto ${ }^{2,3,4}$. 


\section{frecuencia * rango $\cong$ constante}

A fines de los 50, Luhn aplica esta idea a un sistema documental concluyendo, de forma similar, que el poder de resolución (resolving power) deun término de indización asignado en una base de datos, está en los términos de frecuencias intermedias. En este caso, el concepto de poder de resolución de un término - o "peso" como se denominará de aquí en más -, está relacionado con su capacidad de identificar material relevante dentro del

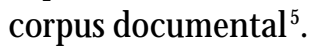

A partir de lostrabajos de Luhn comienzan a desarrollarse funciones matemáticas que modelizan, con mayor rigor, el peso de un término. A Igunas de estas funciones sirven para aplicar en sistemas que utilizan lenguaje controlado y otras para sistemas que emplean la lengua natural. U na de las más simples, y apropiada para la experiencia aquí planteada, fue propuesta por Sparck Jones ${ }^{2,5}$.

$$
\text { Peso del términot }=\log _{2}(\mathbf{n} / \mathbf{f})+1
$$

donde:

- $\mathbf{n}$ es la cantidad de registros de la base de datos

- $\mathbf{f e s ~ l a ~ f r e c u e n c i a ~ d e l ~ t e ́ r m i n o ~ e n ~ l a ~ b a s e ~ d e ~ d a t o s ~}$

Esta función supone que el peso de un término es inversamente proporcional a la cantidad de documentos que lo poseen (véase tabla 1). Puede decirse entonces que, cuanto másfrecuente es un término en una base de datos, menor esla información que proporciona y, por ende, más bajo su peso.

Gerald Salton utiliza este concepto de peso en su modelo de recuperación basado en el espacio vectorial (proyecto SM A RT, 1968). En dicho modelo, se forma una matriz término/documento que representa la base de datos. $\mathrm{C}$ ada vector de la matriz representa un documento; cada elemento del vector tendrá valor 0 (cero) si dicho documento no contiene el término; 0 el valor del peso del término si lo contiene.

V ectorizando a su vez la expresión de búsqueda formulada por el usuario (query vector), desarrolla un nuevo modelo matemático para la recuperación de información basado en el cálculo del coeficiente de similaridad entre vectores. Este coeficiente permite determinar las similitudes y diferencias entre los documentos de una base de datos $y / 0$ entre éstos y la expresión de búsqueda introducida por el usuario.
TABLA 1

\begin{tabular}{ccc}
\hline $\begin{array}{c}\text { (peso alto }=\text { mucha Inf.) } \\
\text { (peso bajo }=\text { poca inf.) }\end{array}$ & $\begin{array}{c}\text { Frecuencia alta } \\
\text { en la base }\end{array}$ & $\begin{array}{c}\text { Frecuencia baja } \\
\text { en la base }\end{array}$ \\
$\begin{array}{c}\text { Frecuencia alta } \\
\text { en el documento }\end{array}$ & Peso medio & Peso alto \\
$\begin{array}{c}\text { Frecuencia baja } \\
\text { en el documento }\end{array}$ & Peso bajo & Peso medio \\
\hline
\end{tabular}

Para realizar el cál culo de la similaridad entre dos vectores existen diversas funciones, siendo las más conocidas la del producto escalar de dos vectores y los coeficientes del coseno, Dice y Jaccard. Para el presente desarrollo se ha seleccionado el coeficiente de D ice, ya que la bibliografía lo presenta como una de las funciones para aplicar en recuperación de información ${ }^{5}$.

Coeficiente de Dice:

$$
\begin{gathered}
2 * \Sigma(\mathbf{P Q} \mathbf{i} * \mathbf{P D i}) \\
\Sigma(\mathbf{P Q} \mathbf{i})+\Sigma(\mathbf{P D} \mathbf{i})
\end{gathered}
$$

Donde:

- PQ i: es el peso del término i en el documento $Q, 00$ (cero) si el documento $Q$ no tiene el término.

- PDi: es el peso del término i en el documento $D, 00$ (cero) si el documento $D$ no tiene el término.

Al hacer el cálculo del coeficiente de similaridad de los documentos y del vector de búsqueda, y someterlos a una comparación sistemática, se está en condiciones de establecer un orden descendente, colocando en primer término el documento cuyo valor es más cercano al del vector de búsqueda y así hasta concluir con todos los registros resultantes. Estos registros son los mismos que se obtienen al hacer un $O R$ entre todos los términos que se utilizan en la interrogación. 


\section{CARACTERÍSTICAS DE LA INTERFACE}

La presente interface ha sido desarrollada con la finalidad de realizar una aplicación concreta de algunos de los principios teóricos de la recuperación de información en forma automática. No obstante, podría aportar en la práctica una posibilidad sustancial de mejorar el acceso a la información de cualquier O PA C sobre plataforma CDS/ ISIS en Internet.

U na de las herramientas que permitela operación de bases M icrolsis desde la W eb es el W W W ISIS 4.0, también conocido como WXIS, desarrollado por el grupo de programación de BIREME. M ediante la interfaz CGI (C ommon $G$ ateway Interface), este software opera como nexo entre las bases de datos y un servidor www, permitiendo la visualización de los registros de las bases ISIS en formato HTM L en el visualizador del cliente.

La solicitud que realiza el cliente se hace a través de un formulario que completa el usuario. Los datos enviados son tomados por el script CGI (en este caso por un script W W W ISIS) que recupera la cadena decaracteresque tiene los datos y la utiliza como expresión de búsqueda en la base de datos. Es el mismo script el que se encarga de devolver los registros resultantes en formato $\mathrm{HTML}$ al cliente.

La interface web desarrollada para este estudio (véase fig. 1) consta de un formulario con las siguientes opciones:

a) el cuadro de texto: donde el usuario registra todos y cada uno de los términos que representan el concepto que está buscando. D ichostérminos se registran uno debajo de otro.

b) porcentajes de similaridad documental: donde el usuario establece el grado de similaridad mínimo entre la información que busca (indicada en los términos ingresados- que componen el vector de búsqueda - ) y los documentos recuperados.

c) campo de la FST : campo de la base de datos sobre el que se efectúa la búsqueda.

d) el botón de Buscar : inicia la acción.

e) el botón Limpiar: borra los datos registrados en el formulario, preparándolo para recibir nueva información.

Un detalle interesante de esta interface es que brinda la posibilidad de que el usuario determine si desea recuperar sólo documentos con un al to porcentaje de similaridad o bien visualizar todos los registros recuperados (listados en orden decreciente según el porcentaje de similaridad).
FIGURA 1

Formulario web diseñado para este estudio

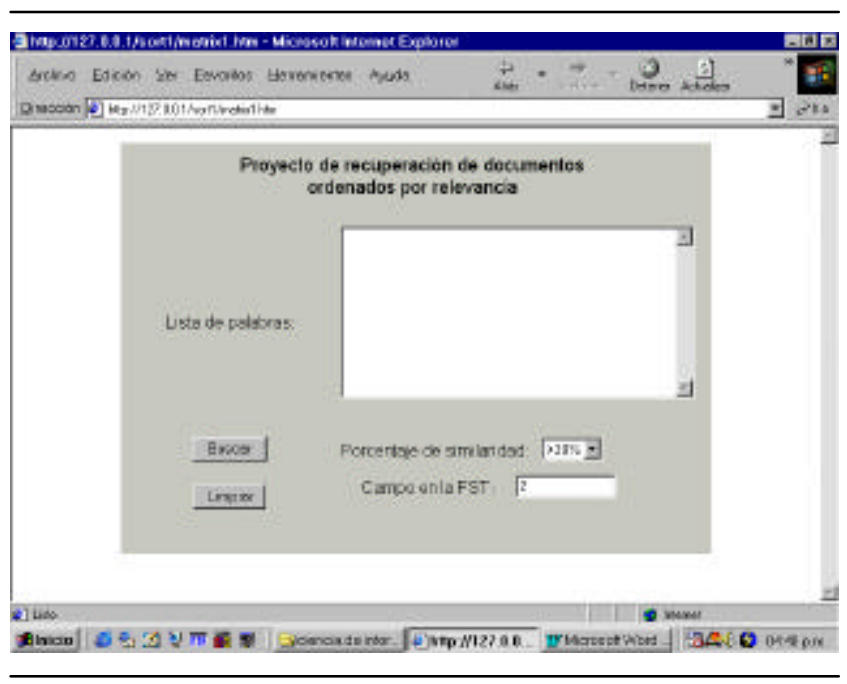

Haciendo una descripción más profunda, debe mencionarse también que fue necesario generar otra base de datos complementaria al OPA C. El ambiente de programación W W W ISIS 4.0 no provee una función que calcule logaritmos, con lo cual el cálculo constante que debe realizarse para conocer el "peso" delostérminoshace poco óptimo el rendimiento de la aplicación. Se optó, entonces, por implementar una tabla de logaritmos (en base DOS) como un archivo maestro ISIS, en el cual se guarde el logaritmo de 1 en el registro 1, el logaritmo de 2 en el registro 2, y así sucesivamente. A dicha base se accede mediantela función REF del lenguaje de formateo.

\section{DESCRIPCIÓN GENERAL DEL SCRIPT}

El funcionamiento de la aplicación comienza cuando el usuario envía los términos a buscar. D ichos términos se utilizan para generar el vector de búsqueda, y además para seleccionar de la base de datos todos los documentos que contienen por lo menos uno de ellos en el campo descriptores.

Luego se toma cada documento recuperado y se calcula la similaridad con el vector de búsqueda mediantela función de similaridad. Si ésta es menor que el mínimo estipulado por el usuario, el documento es descartado; de lo contrario, es insertado en una lista ordenada en forma decreciente de acuerdo al grado de similaridad con el vector de búsqueda. U na vez procesados todos los documentos, son devueltos al usuario. 


\section{G ustavo G abriel A rchuby / Julián C ellini / C laudia Marcela G onzález / M ónica G abriela Pené}

El script consta de un cuerpo principal y dos funciones. U na de las funciones calcula la similaridad entre dos documentos, y la otra, obtiene el peso del término en la base de datos. Esta última función se aplica al campo descriptores de la base de datos, porque se necesita que el término sea único por registro (véase $A$ péndice).

\section{DISCUSIÓN}

La realización de este desarrollo con la única finalidad de profundizar en el estudio del concepto de similaridad documental, permite plantear algunos interrogantes:

1. Si al efectuar búsquedas específicas, con más de 2 términos, no se obtienen resultados numerosos de nuestros OPACs, ¿se justifica el esfuerzo del desarrollo de una interface que los ordene por posible relevancia? Si un resultado de búsqueda ofrece 10 registros para visualizar, ¿es significativo que los devuelva ordenados?

2. Con CDS/ISIS como herramienta de desarrollo reconocida es la potencia de su motor para realizar búsquedas booleanas- , ¿no sería factible obtener resultados ordenados, sin aplicar cálculos de similaridad documental, sólo haciendo variaciones de A ND y OR entre los términos de búsqueda?

3. Losusuarios de nuestros O PA C S, ireclamarán interfaces simples, sin uso de operadores, y resultados "rankeados" tal como los que ofrecen los actuales buscadores de Internet?

Finalmente cabe reflexionar que el presente estudio se enmarca dentro del modelo vectorial, el cual conforma, junto con el modelo probabilístico, el área "dura" de la teoría de recuperación de información. Si partimos de suponer que la búsqueda de información es el problema clave de la Ciencia de la Información, esto implica que el análisis de cualquiera de los temas claves de la Bibliotecología debería plantearse a la luz de sus teorías. Estas teorías provienen de diferentes áreas del conocimiento, principalmente de las que estudian cómo el hombre conoce y procesa información, por lo cual, los modelos matemáticos conforman una pequeña porción dentro de las posibles elecciones para abordar el problema de la recuperación de información.

\section{BIBLIOGRAFÍA}

1. FERNÁN DEZ MOLINA, J. C.; MOYA ANEGÓN, F. de. Los catálogos de acceso público en línea : el futuro de la recuperación de información bibliográfica. Granada : A sociación A ndaluza de bibliotecarios, 1988.

2. MOYA ANEGÓN, F. de Los sistemas integrados de gestión bibliotecaria: estructura de datos y recuperación de información. Madrid : ANABAD, 1995.

3. MOYA ANEGÓN, F. de; LÓPEZ GIJÓN, J.; GARCÍA CARO, C. Técnicas cuantitativas aplicadas a la biblioteconomía y documentación. M adrid : Síntesis, 1996.

4. RIJSBERGEN, C.J. van. Information retrieval. 2. ed. London : Butterworths, 1979.

5. SALTON, G.; MCGILL, M. Introduction to modern information retrieval. N ew York : M cG raw-Hill, 1983. 
Funciones explícitas en el script

\begin{tabular}{|c|c|}
\hline IsisScript & D escripción \\
\hline $\begin{array}{l}<\text { function name }=\text { PesoC ont action }=\text { replace tag }=" 100 "> \\
<\text { do task = keyrange }>\end{array}$ & Encabezado de la función que calcula el peso \\
\hline $\begin{array}{l}<\text { ! - En el campo } 100 \text { sedistinguen lossiguientes subcampos: } \\
\text { ^ ttérmino } \\
\text { ^ b base de datos } \\
\text { ^ } \mathrm{n} \text { tag }->\end{array}$ & $\begin{array}{l}\text { D efinición de constantes y variables necesarias para el cál- } \\
\text { culo del peso. }\end{array}$ \\
\hline 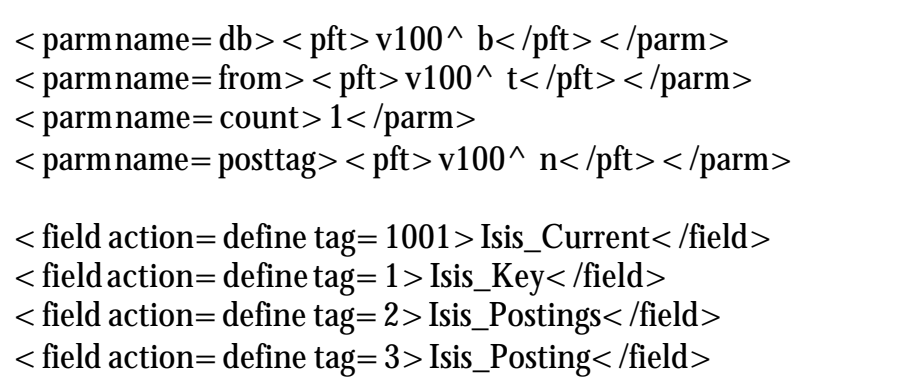 & $\begin{array}{l}\text { Se asigna la } B D\left(v 100^{\wedge} \mathrm{b}\right) \text {, el } \\
\text { Término a recuperar }\left(100^{\wedge} \mathrm{t}\right) \text {. } \\
\text { Identificador del campo Descriptores } \\
\text { En los campos siguientes: } \\
\text { 1= término } \\
2=\text { cantidad de postings del término } \\
3=\text { datos del posting }\end{array}$ \\
\hline & $\begin{array}{l}\text { Cálculo del logaritmo de la cantidad de registros de la base } \\
\text { dedatos. }\end{array}$ \\
\hline $\begin{array}{l}<\text { field action = statusdb tag }=4><\mathrm{pft}>\mathrm{v} 100^{\wedge} \mathrm{b}</ \mathrm{pft} \\
</ \text { field }>\end{array}$ & $\begin{array}{l}\text { Se obtiene información dela BD (campo } 4 \text { subcampo n } \\
=\text { cantidad de registros }+1 \text { ) }\end{array}$ \\
\hline $\begin{array}{l}<\text { field action= replacetag= } 5><\text { pft }> \\
\text { ref(['e:|inetpub|wwwroot|cgi-in|wxis|logs|log2']val(v4^ n)-1,v1) } \\
</ \text { pft }></ \text { field }>\end{array}$ & $\begin{array}{l}\text { Se calcula el logaritmo de la cantidad de registros } \\
\text { (v4^ n) y sele resta 1, guardán dose el resultado en el } \\
\text { campo } 5 \text {. }\end{array}$ \\
\hline $\begin{array}{l}<\text { loop }> \\
<\text { field action }=\text { import tag }=5>5</ \text { field }>\end{array}$ & $\begin{array}{l}\text { C álculo del peso. } \\
\text { v5= Logaritmo de la cantidad de registros }\end{array}$ \\
\hline 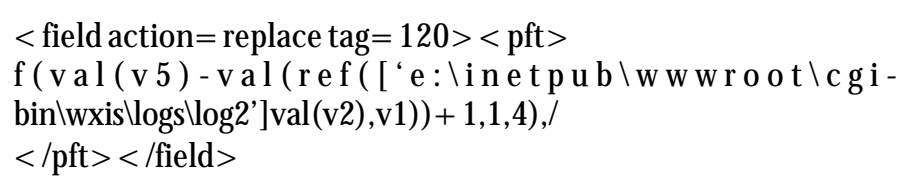 & $\begin{array}{l}\text { La expresión señalada con verde calcula el logaritmo } \\
\text { de la frecuencia del término en la BD. } \\
\text { En el campo } 120 \text { se obtiene el peso que le corresponde } \\
\text { al término en cuestión. }\end{array}$ \\
\hline$<$ field action $=$ exporttag $=120>120</$ field $>$ & \\
\hline$</ l o o p>$ & \\
\hline$</$ do $>$ & \\
\hline $\begin{array}{l}<\text { return action }=\text { exporttag }=100>120</ \text { return }> \\
</ \text { function }>\end{array}$ & $\begin{array}{l}\text { Como resultado dela función se devuelve, en el campo } \\
100, \text { el peso del término. }\end{array}$ \\
\hline
\end{tabular}




IsisScript Descripción
$<$ function name=Simil ice action $=$ replace tag $=" 100 " \quad$ Encabezado de la función quecalcula la similaridad
split $=$ occ $>$

<! - En el campo 100 se distinguen dosocurrencias - una por cada documento a comparar - con loscorrespondientes subcampos:

D efinición de constantes y variables necesarias para el cálculo de la similaridad.

^ d para un término del primer documento

^ q para un término del segundo documento

$\wedge$ b base de datos

^ $\mathrm{t}$ tag en la FST del cual se extrae el término ->

$<$ field action $=$ add tag $=2000><\mathrm{pft}>\left(\mathrm{v} 100^{\wedge} \mathrm{b}\right)</$ pft $></$ field $>$ $<$ field action $=$ add tag $=2004><$ pft $>\left(\mathrm{v100}^{\wedge} \mathrm{t}\right)</ \mathrm{pft}></$ field $>$

Seasignala BD (v100^ b).

$<$ listaction $=$ delete $>$ now $</$ list $>$

$<$ list action $=$ load type $=$ freq $><$ pft $>$ mhu

$\left(\mathrm{v} 100^{\wedge} \mathrm{d} /\right.$ ) $</$ pft $></$ list $>$

Se definen las características de la lista de términos de los documentosa comparar.

$<$ list action $=$ load type $=$ freq $><$ pft $>$ mhu

$\left(\mathrm{v} 100^{\wedge} \mathrm{q} /\right)</ \mathrm{pft}></$ ist $>$

$<$ do task $=$ list $>$

$<$ parm name $=$ reverse $>0 \mathrm{n}</$ parm $>$

$<$ parm name $=$ sort $><$ pft $>\mathrm{f}($ val $($ v2 $), 10,0)</$ pft $></$ parm $>$

$<$ field action $=$ define tag $=1001>\mid$ sis_Current $</$ field $\rangle$

$<$ field action $=$ definetag $=1003>\mid$ sis_ ${ }_{-}$Total $\mid</$field $>$

$<$ field action = define tag $=1>$ Isis_ $\mid$ tem $</$ field $>$

$<$ field action $=$ define tag $=2>\mid$ sis_ $V$ alue $</$ field $>$

$<$ field action $=$ replace tag $=1101>0</$ field $>$

$<$ field action $=$ replace tag $=1102>0</$ field $>$

$<$ loop $>$

$<$ ! - N ombre dela base de datos $->$

$<$ field action $=$ import tag $=2000>2000</$ field $>$

$<$ ! - T ag de la Fst del que se extrae el término - >

$<$ field action = import tag $=2004>2004</$ field $>$

$<$ ! - Campo dondese genera el divisor $->$

$<$ field action = import tag $=1101>1101</$ field $>$

$<$ !-Campo donde se genera el dividendo- $>$

$<$ field action = import tag $=1102>1102</$ field $>$
G eneración dela lista

Segenera una lista que contiene lostérminos de ambosdocumentos.

$$
\begin{aligned}
& 2 * \Sigma(\mathrm{PQi} * \mathrm{PDi}) \quad \text { Dividendo } \\
& ------------ \\
& \Sigma(\mathrm{PQ} \mathrm{i})+\Sigma(\mathrm{PDi}) \quad \text { Divisor }
\end{aligned}
$$

En el campo 1101 se genera el dividendo.

En el campo 1102 se genera el divisor.

Lasinstrucciones encerradas entre $<100 p>$ y

$</ l o o p>$ se repiten para cada elemento dela lista. 


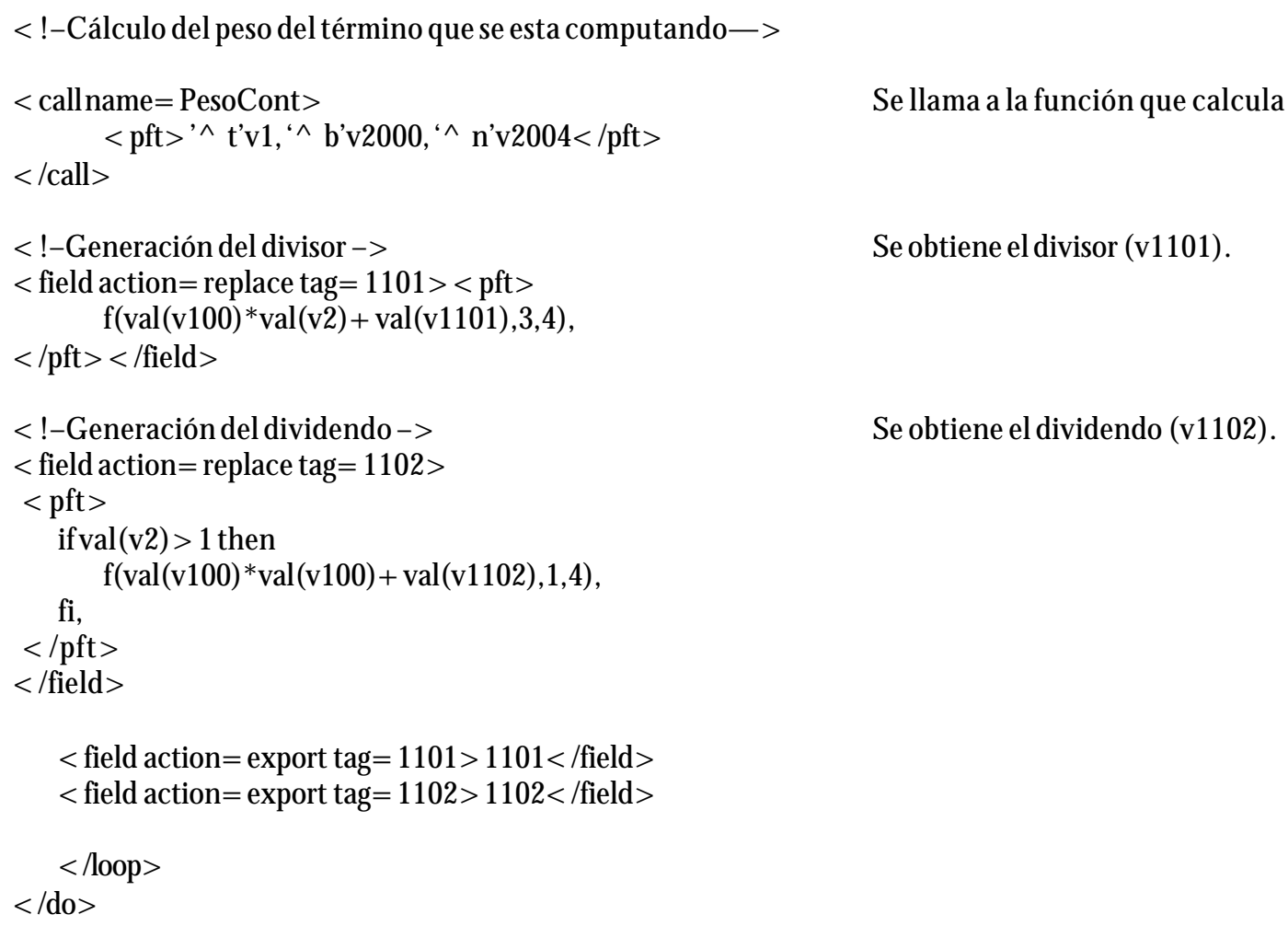

Se obtieneel divisor (v1101).

Se obtiene el dividendo (v1102).

Sellama a la función que calcula el peso del término. 
C uerpo principal del script

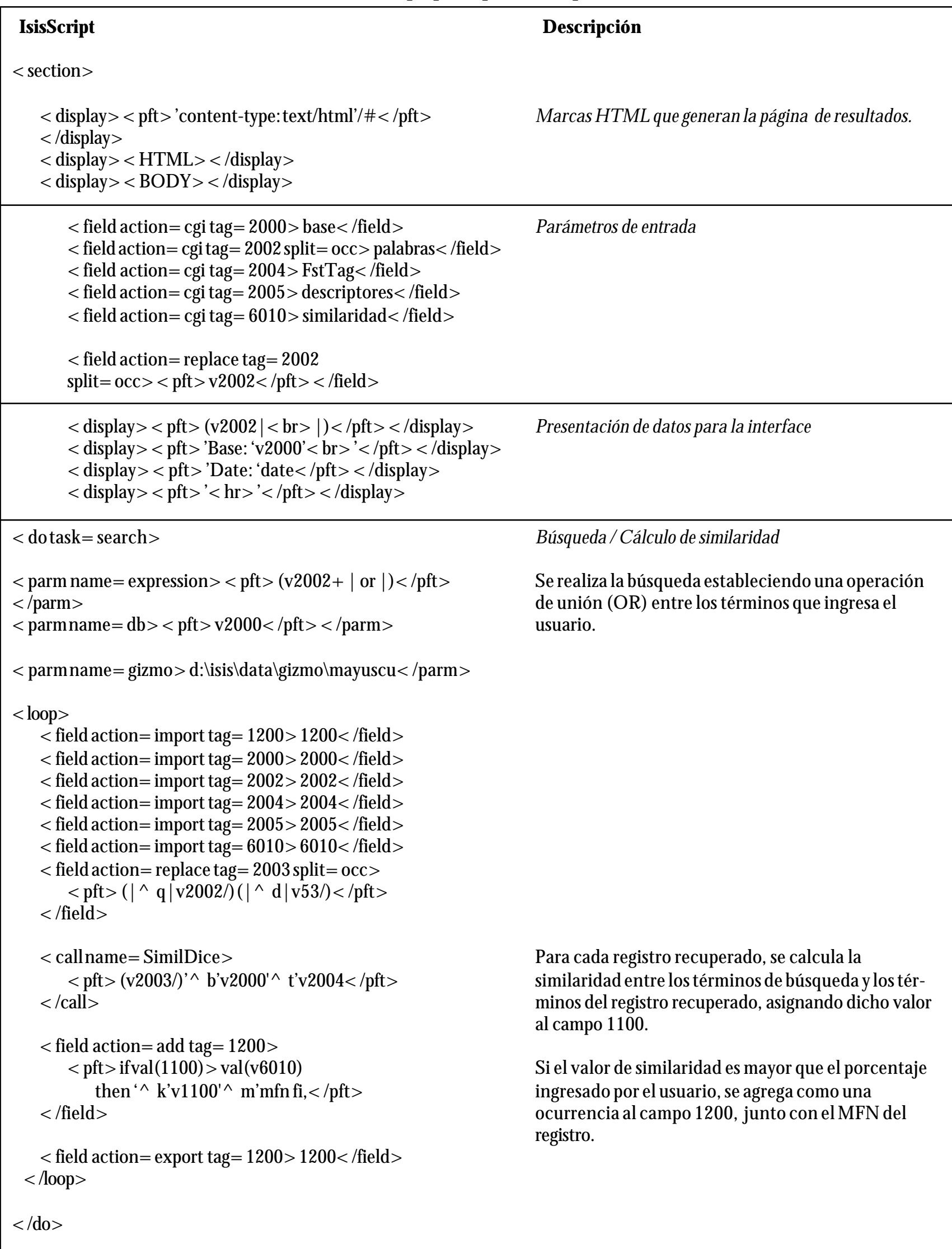




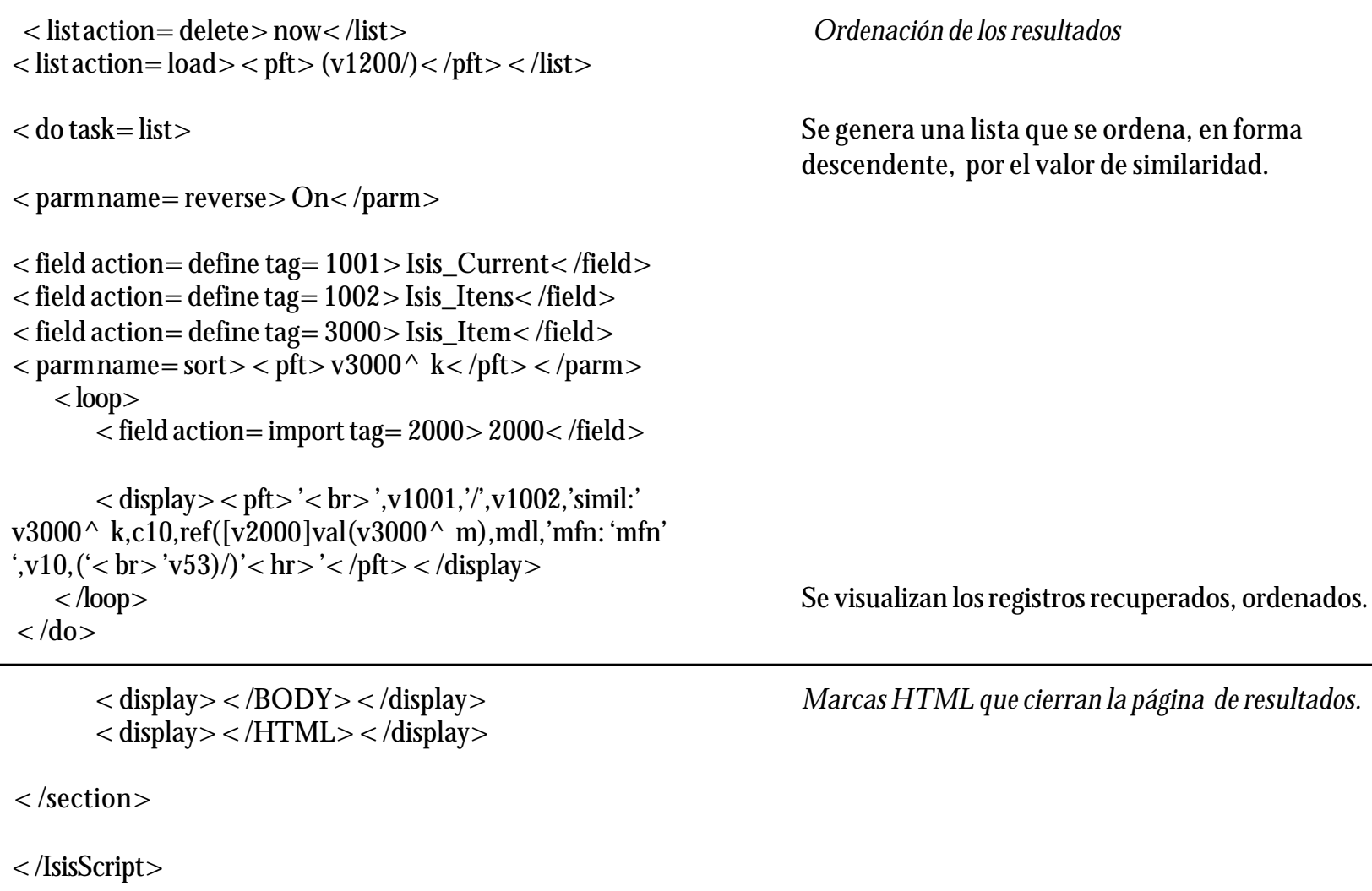

M arcas H T M L quecierran la página de resultados.

\section{O rdenación delos resultados}

Se visualizan los registros recuperados, ordenados. 